\title{
Interações entre medicamentos sujeitos a controle especial dispensados na Farmácia Distrital Centro de Porto Alegre, RS, Brasil
}

\author{
Interactions among medicines under special control in the District \\ Pharmacy Centro in Porto Alegre, RS, Brazil
}

Recebido em: 23/03/2018

Aceito em: 23/08/2018
Adriane PASQUALOTTO ${ }^{1}$; Paula Eliete Rodrigues BITENCOURT ${ }^{2}$; Natália Azuaga NIETIEDT ${ }^{1}$; Leonardo Fernandes de PAULA ${ }^{1}$; Rafael de Oliveira NOGUEIRA ${ }^{3}$; Rosane GOMEZ ${ }^{2}$

${ }^{1}$ Faculdade de Farmácia, Universidade Federal do Rio Grande do Sul. Av. Ipiranga, 2752, Azenha, CEP 90610-000. Porto Alegre, RS, Brasil. ${ }^{2}$ Programa de Pós-Graduação em Farmacologia e Terapêutica, Instituto de Ciências Básicas da Saúde, Campus Centro, Universidade Federal do Rio Grande do Sul, Rua Sarmento Leite, 500, sala 305, CEP 90050-170. Porto Alegre, RS, Brasil. ${ }^{3}$ Centro de Saúde Santa Marta, Prefeitura Municipal, R. Cap. Montanha, 27, Centro, 90010-040.Porto Alegre, Brasil.E-mail: bitencourt.paula@yahoo.com.br

\section{ABSTRACT}

Polytherapy is a useful practice in the treatment of coexisting conditions or to enhance the pharmacological effect in less responsive conditions, but it presents a significant risk for drug interactions. Thus, the objective of this study was to identify in prescriptions issued by the Farmácia Distrital Centro in Porto Alegre, RS, possible drug interactions of medicines under special control and to classify them about potential drug interactions and degree of severity using the database MICROMEDEX ${ }^{\circledR}$. After analyzing 2,839 prescriptions, the results showed that the majority of the patients were female and $75 \%$ of prescriptions presented some interaction. Out of 291 drug-drug associations analyzed. $44.4 \%$ gave a substantial risk of interaction, 23.9\% moderate risk and 6.5\% minor risk. Most of the strong-risk interactions showed cardiotoxicity as the main risk. The high frequency of interactions and the risk of toxic effects, also point to the need for an adequate information system on the use of medications as well as pharmaceutical guidance in these health facilities.

Keywords: prescriptions; psychotropics; health center; pharmaceutical attention; risk

\section{RESUMO:}

A politerapia é uma prática útil no tratamento de patologias coexistentes ou para potencializar o efeito farmacológico em condições pouco responsivas, porém apresenta um grande risco para interações medicamentosas. Sendo assim, o objetivo deste estudo foi identificar em prescrições atendidas pela Farmácia Distrital Centro em Porto Alegre, RS, as interações medicamentosas de medicamentos sujeitos a controle especial e classificá-las quanto a potenciais interações medicamentosas e grau de severidade com auxílio da base de dados MICROMEDEX ${ }^{\circledR}$. Após análise de 2.839 receituários, os resultados mostraram que grande parte dos pacientes pertencia ao sexo feminino e que $75 \%$ do total das prescrições apresentavam algum tipo de interação. Das 291 associações analisadas, 44,4\% apresentavam risco forte de interação, $23,9 \%$ risco moderado e $6,5 \%$ risco leve. A maioria das interações com risco forte apresentava como principal risco a cardiotoxicidade. A elevada frequência de interações e o risco de efeitos tóxicos, aponta para a necessidade de um sistema adequado de informações sobre o uso de medicamentos e de orientação farmacêutica nessas unidades de saúde.

Palavras-chave: prescrições; psicotrópicos; centro de saúde; atenção farmacêutica; risco. 


\section{INTRODUÇÃO}

A polifarmácia é caracterizada pela associação de medicamentos administrados em um mesmo paciente e é uma prática comum em consultórios e hospitais (1). Essa prática se justifica pelo diversificado número de doenças apresentadas por um único indivíduo, sendo útil no tratamento das comorbidades e, também, quando outros fármacos são adicionados à terapia de base com a intenção de melhorar os efeitos dos demais $(2,3)$. No entanto, a politerapia está diretamente associada ao aumento de custos assistenciais, atendimentos de emergência e internações hospitalares, além de ser fator de risco para reações adversas e interações medicamentosas (4-6).

Interação medicamentosa pode ser conceituada como alteração dos efeitos de um fármaco devido à administração simultânea de outro fármaco (7). Os mecanismos pelos quais as interações podem ocorrer são variados e complexos e podem afetar tanto parâmetros farmacocinéticos quanto farmacodinâmicos que resultam não somente em complicações fisiológicas, mas também em prejuízo econômico para os pacientes e para a sociedade $(8,9)$. De fato, a administração concomitante de dois ou mais medicamentos pode afetar a absorção, distribuição, metabolismo ou excreção de um deles, de maneira a reduzir sua biodisponibilidade e promover a potencialização ou anulação do efeito terapêutico esperado (10). Vários fatores relacionados ao paciente, ao fármaco e à prescrição médica podem potencializar o risco de interações, além de fatores ambientais e genéticos $(11,12)$.

O grau de risco para uma dada interação medicamentosa está diretamente relacionado à sua capacidade de afetação dos sistemas (13). Dentre os grupos farmacológicos que mais apresentam risco de interação estão aqueles que atuam no sistema nervoso central como ansiolíticos, antidepressivos antiepiléticos, antipsicóticos e outros (10). A dispensação desses medicamentos sujeitos a controle especial exige Receituário de Controle Especial ou Notificação de Receita, sendo incluídos na Portaria 344/98 da Agência Nacional de Vigilância Sanitária (Anvisa) (14). Muitos desses medicamentos constam da Relação Municipal de Medicamentos Essenciais (REMUME) (15) e estão disponíveis para dispensação gratuita aos usuários do Sistema Único de Saúde (SUS) pelas farmácias distritais.

O crescente número de internações devido a interações medicamentosas e os gastos a elas relacionados estimulam a busca de alternativas que possam melho- rar a segurança e qualidade de vida dos pacientes, bem como o controle dos custos em saúde. No Brasil, o elevado número de usuários do SUS nos locais de dispensação gera um grande número de receituários atendidos regularmente, o que inviabiliza a análise criteriosa pelo farmacêutico na identificação de possíveis interações medicamentosas. Sendo assim, este estudo tem como objetivo identificar interações medicamentosas em prescrições de medicamentos sujeitos a controle especial dispensados pela Farmácia Distrital Centro, do Centro de Saúde Santa Marta (FDC/CSSM), em Porto Alegre, RS e classificá-las quanto ao risco utilizando uma base de dados amplamente empregada em serviços de atenção farmacêutica denominada MICROMEDEX ${ }^{\circledR}$.

\section{MÉTODOS}

Este foi um estudo observacional transversal desenvolvido a partir da seleção de receituários de medicamentos sujeitos a controle especial pela portaria 344/98 da Anvisa (14) que compõem a REMUME de Porto Alegre, RS (15), de pacientes atendidos na Farmácia Distrital Centro, do Centro de Saúde Santa Marta (FDC/CSSM), em Porto Alegre, RS, durante o mês de novembro de 2011.

Para esse estudo foram selecionadas prescrições de pacientes que utilizavam mais de um medicamento sujeito a controle especial, sendo incluídas as prescrições com dois ou mais medicamentos em um mesmo receituário e/ou dois ou mais medicamentos em mais de um receituário prescrito para o mesmo paciente. Foram consideradas variáveis como sexo, inferida pelo nome do paciente, origem da prescrição e medicamentos utilizados pelo paciente. Cabe ressaltar que foram avaliadas apenas as prescrições, sem seguimento do efeito terapêutico ou efeitos adversos causados pelo uso dos medicamentos dispensados. Foram considerados critérios de exclusão aquelas receitas que apresentavam apenas um medicamento sujeito a controle especial, indicando uso de psicotrópicos como monoterapia.

Para avaliação das interações medicamentosas entre os medicamentos controlados, foi utilizada a base de dados MICROMEDEX ${ }^{\circledR}$ (16), sendo identificadas e classificadas quanto ao grau de risco para cada associação. Nesta base de dados, grau de risco forte é considerado aquele que potencialmente ameaça a vida ou é capaz de causar dano permanente (17). Risco moderado é quando a interação promove risco de prejuízo à saúde do paciente, ou quando exige tratamento adicional, 
hospitalização ou aumento do período de internação. Risco leve são aquelas interações cujos efeitos podem ser incômodos ou não afetam de modo significativo o efeito esperado da terapia, sem necessidade de tratamento adicional (17). Os dados obtidos foram analisados e comparados com a literatura.

O projeto foi aprovado pelo Comitê de Ética em Pesquisa da Prefeitura Municipal de Porto Alegre- RS, conforme processo $\mathrm{n}^{\circ}$ 001.038963.11.6.

\section{RESULTADOS E DISCUSSÃO}

Os resultados mostraram que do total de pacientes, $59,5 \%$ eram do sexo feminino e $40,5 \%$ eram do sexo masculino. Esses resultados corroboram com outros estudos realizados em diferentes regiões no Brasil que mostraram que as mulheres ( $55 \%$ a $80 \%)$ são as principais usuárias de psicotrópicos (18-20). Além disso, aparentemente, o sexo feminino apresenta um risco maior de distúrbios de humor quando comparado com o sexo masculino, pois as variações hormonais sexuais parecem influenciar a modulação do sistema neuroendócrino feminino (20-22). Outro fato que pode estar associado a esse resultado é que as mulheres apresentam uma maior preocupação com a sua condição de saúde e, consequentemente, frequentam mais os serviços de saúde.

Quanto ao local de origem dos receituários, grande parte era oriunda do próprio Centro de Saúde Santa Marta, seguido pelo Hospital de Clínicas de Porto Alegre (HCPA) e hospitais do Grupo Hospitalar Conceição, também de Porto Alegre. Esse resultado pode ser devido ao fato de que o Centro de Saúde Santa Marta oferece diversas especialidades médicas e atende cerca de 240 mil usuários SUS, de acordo com a Prefeitura Municipal de Porto Alegre.

A análise das prescrições permitiu identificar que as classes farmacológicas mais prescritas para os usuários da FDC/CSSM foram os antidepressivos, seguidos pelos anticonvulsivantes e antipsicóticos. Destaca-se que a frequência de receituários com indicação dessas classes de fármacos pode ter sido ainda maior, pois foram excluídos deste estudo aqueles que os utilizavam como monoterapia. Dentre os três medicamentos com maior frequência de dispensação encontram-se a fluoxetina (20 mg), presente em mais de $36 \%$ dos receituários, a clorpromazina em suas diversas apresentações (comprimidos de $25 \mathrm{mg}$ e $100 \mathrm{mg}$, e solução oral de $40 \mathrm{mg} /$ $\mathrm{mL}$ ), prescrito em $30 \%$ dos receituários, seguido pela carbamazepina em suas apresentações (comprimidos de $200 \mathrm{mg}$ e suspensão oral de $20 \mathrm{mg} / \mathrm{mL}$ ), perfazendo mais de $28 \%$ das prescrições (Tabela 1 ).

Tabela 1. Frequência de prescrição de medicamentos sujeitos a controle especial dispensados Farmácia Distrital Centro, no Centro de Saúde Santa Marta, Porto Alegre- RS, no período de Novembro de 2011.

\begin{tabular}{|l|c|}
\hline Medicamento & prescrições (\%) \\
\hline Fluoxetina & 36,92 \\
\hline Clorpromazina & 30,06 \\
\hline Carbamazepina & 28,32 \\
\hline Amitriptilina & 27,95 \\
\hline Haloperidol & 23,07 \\
\hline Carbonato de Lítio & 20,53 \\
\hline Biperideno & 20,47 \\
\hline Diazepan & 20,47 \\
\hline Ácido Valpróico & 15,89 \\
\hline Imipramina & 12,31 \\
\hline Fenobarbital & 8,97 \\
\hline Fenitoína & 4,51 \\
\hline
\end{tabular}

Mota e Silva Jr. (2012) mostraram que o consumo de fluoxetina é considerado alto no Rio Grande do Sul, chegando a $58 \mathrm{mg} /$ per capita (23). Isto pode ser justificado pela alta prevalência de indivíduos diagnosticados com depressão nessa região (24). Outro fato que também pode contribuir para essa alta frequência de uso é que a fluoxetina apresenta poucos efeitos adversos quando comparado às demais classes de antidepressivos, como os tricíclicos também disponibilizados pelo SUS.

Das 291 combinações identificadas, a associação mais frequente ocorreu entre os medicamentos fluoxetina e amitriptilina (Tabela 2), apoiando os estudos descritos acima a respeito da ampla utilização da fluoxetina. Entretanto, a associação de um inibidor seletivo da recaptação de serotonina com um antidepressivo tricíclico apresenta um risco maior de interação, pois, associado à sua baixa seletividade, o uso concomitante pode resultar na toxicidade dos antidepressivos tricíclicos. Além de efeitos adversos como boca seca, retenção urinária e sedação, é descrito o aumento do risco de cardiotoxicidade, devido ao prolongamento do intervalo QT para essa associação (16). Entretanto, deve ser considerado que a combinação da terapia com fluoxetina e antidepressivo tricíclico pode ser usada para o tratamento de depressão resistente, caracterizando um caso de potencialização medicamentosa em que o paciente necessitaria ser 
monitorado (17). Ainda, a utilização da associação entre fluoxetina e amitriptilina também é prescrita para outras patologias como dor crônica, enxaqueca, entre outros (13). Entretanto, vale ressaltar que prescrições de me- dicamentos não psicotrópicos, interações entre medicamentos psicotrópicos e outros não sujeitos à retenção de receita, bem como as interações desejadas pelo prescritor não foram avaliadas nesse estudo.

Tabela 2. Pacientes com prescrição de risco forte, tipo de associação, distribuição de risco e efeito tóxico observado pela interação entre os fármacos dispensados na Farmácia Distrital Centro, no Centro de Saúde Santa Marta, Porto Alegre, RS, em Novembro de 2011.

\begin{tabular}{|clcl}
\hline Frequência & Fármaco 1 & Fármaco 2 & \\
\hline 234 & Amitriptilina & Fluoxetina & Cardiotoxicidade (prolonga intervalo QT, parada cardíaca) \\
\hline 145 & Clorpromazina & Carbonato de Lítio & Fraqueza,discinesias, $\uparrow$ dos sintomas extrapiramidais, encefalopatia e danos cerebrais \\
\hline 120 & Clorpromazina & Holoperidol & Cardiotoxicidade (prolonga intervalo QT, parada cardíaca) \\
\hline 104 & Holoperidol & Carbonato de Lítio & Fraqueza,discinesias, $\uparrow$ dos sintomas extrapiramidais, encefalopatia e danos cerebrais \\
\hline 87 & Clorpromazina & Fluoxetina & Cardiotoxicidade (prolonga intervalo QT, parada cardíaca) \\
\hline 73 & Amitriptilina & Clorpromazina & Cardiotoxicidade (prolonga intervalo QT, parada cardíaca) \\
\hline 66 & Amitriptilina & Haloperidol & Cardiotoxicidade (prolonga intervalo QT, parada cardíaca) \\
\hline 64 & Clorpromazina & Imipramina & Cardiotoxicidade (prolonga intervalo QT, parada cardíaca) \\
\hline 62 & Haloperidol & Fluoxetina & $\uparrow$ toxicidade do haloperidol (pseudoparkinsonism, acatisia, rigidez) e $\uparrow$ riscode cardiotoxicidade \\
\hline 51 & Fluoxetina & Imipramina & $\uparrow$ toxicidade dos ADT (boca seca, retenção urinária, sedação) e $\uparrow$ risco decardiotoxicidade \\
\hline 15 & Amitriptilina & Imipramina & Cardiotoxicidade (prolonga intervalo QT, parada cardíaca) \\
\hline 11 & Fenobarbital & Diazepam & $\uparrow$ Risco de depressão respiratória \\
\hline
\end{tabular}

ADT: antidepressivo tricíclico; $\uparrow:$ aumenta

Interessantemente, além da interação com a fluoxetina, a amitriptilina está presente em outros quatro tipos de associações classificadas como risco forte. Algumas dessas combinações não apresentam justificativa farmacológica para seu uso, como a associação entre amitriptilina e imipramina, uma vez que ambas pertencem à classe de antidepressivos tricíclicos e podem ter seus efeitos adversos potencializados pelo uso concomitante. Os profissionais de saúde devem ser alertados sobre as interações que apresentam risco de prolongamento do intervalo QT e sobre aquelas que promovem arritmias, para que assim possam monitorar esses efeitos ou até mesmo substituir estes medicamentos. Dessa maneira, a utilização de bases de dados como o MICROMEDEX ${ }^{\circledR}$, instrumento tecnológico empregado neste estudo para identificar risco de interações pelo uso de mais de um psicotrópico, podem auxiliar na fase de dispensação de medicamentos para usuários de centros de atendimento à saúde, como o FDC/CSSM, estabelecendo uma relação direta entre o uso racional de medicamentos, segurança e redução de custos, seja com o próprio medicamento ou internações advindas de interações medicamentosas.

Estudos realizados em ambiente hospitalar mostraram que quanto maior o número de medicamentos, maior a chance de interações e, consequentemente, a severidade dos riscos dessas (25). Esse quadro parece se repetir também na dispensação em farmácias distritais, pois foi possível verificar que $75 \%$ das prescrições apresentavam algum tipo de interação, sendo que $44 \%$ dessas foram classificadas como de risco forte pela base de dados MICROMEDEX ${ }^{\circledR}$, como mostrado na Figura 1.

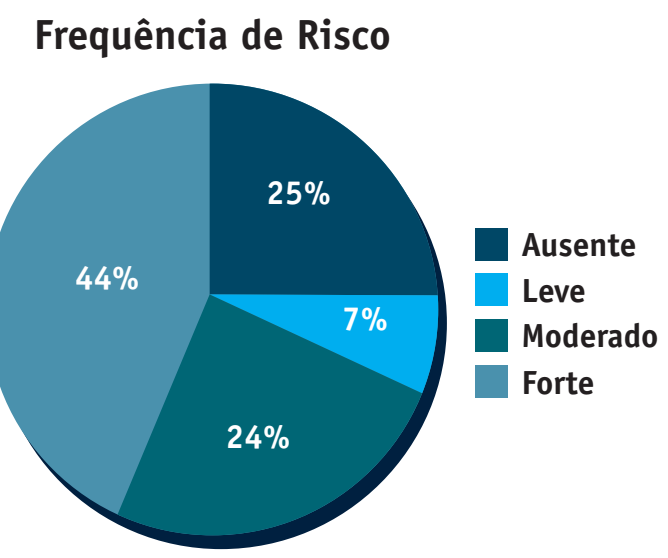

Figura 1. Classificação de interações medicamentosas quanto ao grau de risco, identificado em receituários de medicamentos sujeitos a controle especial dispensados Farmácia Distrital Centro, no Centro de Saúde Santa Marta, Porto Alegre- RS, no período de Novembro de 2011. 
Os resultados encontrados nesse trabalho vão de encontro ao estudo realizado no HCPA, onde $28 \%$ do total de interações medicamentosas identificadas pelo Centro de Informações sobre Medicamentos (CIM) foram classificadas como risco forte (26). A evidenciada discrepância entre as frequências de interações de risco forte pode ser explicada pelas diferenças entre os locais de investigação, uma vez que hospitais possuem equipes multidisciplinares com acesso a programas informatizados e protocolos que identificam as possíveis interações no momento da prescrição ou dispensação, além da presença de profissional farmacêutico para monitoramento das prescrições, o que não acontece em sua totalidade nas farmácias distritais.

\section{CONCLUSÃO}

A elevada ocorrência de interações observadas no presente estudo ressalta a importância da avalia- ção das prescrições, tanto por parte do médico, como por parte do farmacêutico em farmácias distritais, como a FDC/CSSM. É indispensável uma maior e melhor articulação entre as equipes multidisciplinares para avaliar, criteriosamente, a racionalidade de esquemas terapêuticos, detectar as interações medicamentosas e assim melhorar a qualidade de vida dos pacientes. A utilização de bases de dados confiáveis e de fácil compreensão, como o MICROMEDEX ${ }^{\circledR}$, pode auxiliar não somente a identificação, mas também a avaliação dos riscos de interações medicamentosas. Entretanto, se faz necessária a realização de mais estudos que considerem o acompanhamento de pacientes usuários de medicamentos sujeitos a controle especial e estudos de farmacovigilância para uma maior compreensão sobre impacto dessas interações medicamentosas.

\section{REFERÊNCIAS}

1. Morin L, Johnell K, Laroche ML, Fastbom J, Wastesson JW. The epidemiology of polypharmacy in older adults: register-based prospective cohort study.Clin. Epidemiol. 2018;12(10):289-298. DOI: 10.2147/CLEP.S153458

2. Duerden M, Avery T, Payne R. Polypharmacy and medicines optimisation. making it safe and sound. London: The King's Fund; 2013.

3. Scott IA, Hilmer SN, Reeve E, et al. Reducing inappropriate polypharmacy: the process of deprescribing. JAMA Intern. Med. 2015;175(5):827-834. DOI: 10.1001/jamainternmed.2015.0324

4. Davies EA, O’Mahony MS. Adverse drug reactions in special populations - the elderly. Braz. J. Clin. Pharmacol. 2015;80(4):796-807. DOI: 10.1111/bcp.12596

5. Wauters M, Elseviers M, Vaes B, Degryse J, Dalleur O, Stichele RV, Christiaens T, Azermai M. Too many, too few, or too unsafe? Impact of inappropriate prescribing on mortality, and hospitalization in a cohort of community-dwelling oldest old. Braz. J. Clin. Pharmacol. 2016;82(5):1382-1392. DOI: 10.1111/bcp.13055

6. Secoli SR. Polifarmácia: interações e reações adversas no uso de medicamentos por idosos. Rev. Bras. Enf. 2010;63(1):136-140. DOI: $10.1590 / \mathrm{S} 0034-$ 71672010000100023

7. Lima RE, Cassiani SH. Interações medicamentosas potenciais em pacientes de unidade de terapia intensiva de um hospital universitário. Rev. Latinoam. Enferm. 2009;17(2):81-87. DOI: 10.1590/S010411692009000200013
8. Fried TR, O'Leary J, Towle V, Goldstein MK, Trentalange M, Martin DK. Health outcomes associated with polypharmacy in community dwelling older adults: a systematic review. J. Am. Geriatr. Soc. 2014;62(12):22612272. DOI: $10.1111 /$ jgs. 13153.

9. Franchi C, Marcucci M, Mannucci PM, Tettamanti M, Pasina L, Fortino I, Bortolotti A, Merlino L, Nobili A. Changes in clinical outcomes for community-dwelling older people exposed to incident chronic polypharmacy: a comparison between 2001 and 2009. Pharmacoepidemiol. Drug Saf. 2016;25(2):204-11. DOI: 10.1002/ pds.3938

10. Wu B, Bruns EJ, Tai MH, Lee BR, Raghavan R, Reis S. Psychotropic polypharmacy among youths with serious emotional and behavioral disorders receiving coordinated care services. Psychiatr. Serv.2018; 15:appips201700357. DOI: 10.1176/appi.ps.201700357.

11. Zeeh J. Polypharmacy - a description which doesn't give much useful information? MMW Fortschr Med. 2018;160(5):38-41. DOI: 10.1007/s15006-018-0288-4.

12. Santanasto AJ, Goodpaster BH, Kritchevsky SB, Miljkovic I, Satterfield S, Schwartz AV, Cummings SR, Boudreau RM, Harris TB, Newman AB. Body composition remodeling and mortality: the health aging and body composition study. J. Gerontol. A Biol. Sci. Med. Sci. 2017;72(4):513-519. DOI: 10.1093/gerona/glw163.

13. Brunton LL, Lazo JS, Parker KL. Goodman \& Gilman. As bases Farmacológicas da Terapêutica. Editora McGraw-Hill, $11^{\circ}$ Ed. 2010. 
14. BRASIL. Ministério da Saúde. Portaria 344, de 12 de maio de 1998. Aprova o regulamento sobre substâncias e medicamentos sujeitos a controle especial. Diário Oficial da União 1999; (21):29-53.Seção 1. Acesso em 28/02/2018.

15. SMS. REMUNE. Relação Municipal de Medicamentos Essenciais. Prefeitura Municipal de Porto Alegre. Secretaria Municipal de Saúde, 2006. Acesso em 28/02/2018.

16. Micromedex ${ }^{\circledR}$ Healthcare Series. Thomson. Base de Dados. Disponível em: http://www.thomsonhccom.ez45. periodicos.Capes.gov.br/micromedex2/librarian/ND_T/ evidencexpert/ND_PR/evidencexpert/CS/973879/ND_ AppProduct/evidencexpert/DUPLICATIONSHIELDSYNC/CCF3FF/ND_PG/evidencexpert/ND_B/evidencexpert/ND_P/evidencexpert/PFActionId/evidencexpert. Find DrugInteractions. Acesso em 15 de março de 2012.

17. Tatro DS. Drug interaction facts: the authority on drug interactions. Wolters Kluwer Health. 2010; 2035p.

18. Campigotto KF, Teixeira JJ, Cano FG, Sanches AC, Cano MF, Guimarães DS. Detecção de risco de interações entre fármacos antidepressivos e associados prescritos a pacientes adultos. Rev. Psiquiatr. Clín. 2008;35(1):1-5. DOI: 10.1590/S0101-60832008000100001.

19. Santos HC, Ribeiro RR, Ferrarini M, Fernandes JPS. Possíveis interações medicamentosas com psicotrópicos encontradas em pacientes da Zona Leste de São Paulo. Rev. Cienc. Farm. Básica Apl. 2009;30(3):285-289.

20. Menezes FG, Mariz LCV. Interações medicamentosas e efeitos adversos que ocorrem em prescrições do SUS com o medicamento fluoxetina no Distrito de São Paulo, Zona Norte. Rev Eletr Farmácia. 2012;9(1):1-17. DOI: 10.15448/1983-652X.2015.3.21093

21. McGregor C, Riordan A, Thornton J. Estrogens and the cognitive symptoms of schizophrenia: possible neuroprotective mechanisms. Front. Neuroendocrinol. 2017;47:19-33. DOI: 10.1016/j.yfrne.2017.06.003.

22. Seeman MV, González-Rodríguez A. Use of psychotropic medication in women with psychotic disorders at menopause and beyond. Curr. Opin. Psychiatry. 2018. DOI: 10.1097/YCO.0000000000000410.

23. Mota DM, Silva-Jr GG. Evidências advindas do consumo de medicamentos moduladores do apetite no Brasil: um estudo farmacoeconométrico. Rev. Assoc. Med. Bras. 2012;58(1):88-94. DOI: 10.1590/S010442302012000100020.

24. IBGE. Instituto Brasileiro de Geografia e Estatística. Disponível em: https://ww2.ibge.gov.br/home/estatistica/ populacao/trabalhoerendimento/pnad98/saude/metodologia.shtm. Acesso em 18/03/2018. 